\title{
Trends in Moderate Rainfall Extremes: A Regional Monotone Regression Approach
}

\author{
M. RoTH \\ Royal Netherlands Meteorological Institute (KNMI), De Bilt, and EURANDOM, Eindhoven University \\ of Technology, Eindhoven, Netherlands \\ T. A. BUISHAND \\ Royal Netherlands Meteorological Institute (KNMI), De Bilt, Netherlands \\ G. JONGBLOED \\ Delft Institute of Applied Mathematics, Delft University of Technology, Delft, Netherlands
}

(Manuscript received 10 October 2014, in final form 6 July 2015)

\begin{abstract}
Rainfall extremes are thought to have increased over recent years. Typically linear trends have been considered to describe the temporal evolution of high quantiles of the daily rainfall distribution. For long records it is important to allow more flexibility. Quantile regression methods are available to estimate monotone trends for single stations. Having multiple stations in a region, the significance of the trend at the regional scale is often of interest. From this perspective the authors propose a regression approach that can be used to estimate a common monotone trend for the site-specific quantiles. Moreover, the method allows for the construction of confidence bands and testing the hypothesis of an existing nondecreasing trend against the null hypothesis of no trend. The approach is applied to 102 series of daily rainfall over the Netherlands for the period 1910-2009. The results are compared with those from a (regional) Mann-Kendall test. Significantly increasing trends are found for the winter season and for the whole year. In the summer season trends are less consistent over the region and are only significant in the western part of the Netherlands. For the summer season, linearity of the trend seems less apparent than for winter and for the whole year. However, the deviation from linearity is not significant.
\end{abstract}

\section{Introduction}

Changes in the occurrence of heavy precipitation may have serious societal impacts. The implementation of adaptation measures depends on the statistical significance of observed changes. Many researchers have tested the significance of changes in moderate rainfall extremes, using indices such as the number of days exceeding a specific threshold (e.g., $20 \mathrm{~mm}$ ) or annual/ seasonal quantiles (Klein Tank and Können 2003; Moberg and Jones 2005; Alexander et al. 2006). Quantiles refer to the same part of the distribution everywhere and are therefore more suitable for spatial comparison than counts of exceedances of an absolute

Corresponding author address: M. Roth, Royal Netherlands Meteorological Institute (KNMI), P.O. Box 201, 3730 AE, De Bilt, Netherlands.

E-mail: roth@knmi.nl threshold (Klein Tank and Können 2003). Linear regression has often been used to test for changes in sample quantiles. A difficulty with this approach is that high quantiles from small samples can be seriously biased. Quantile regression can be used to overcome this difficulty (Wasko and Sharma 2014).

Linear trend modeling is typically used because of its broad acceptance (cf. Hartmann et al. 2013, Box 2.2). However, when considering long measurement records, with possible phases of stagnation, linear modeling is not flexible enough (see e.g., Villarini et al. 2011). The MannKendall (MK) test can be used to test the hypothesis of a monotonic trend in the annual or seasonal quantiles against the null hypothesis of no trend. However, it does not provide a visualization of the trend itself.

Monotone quantile regression provides an alternative that does not rely on the biased annual quantiles and provides a visualization of the trend. Monotone trends can be estimated by order restricted regression (Robertson 
et al. 1988). While order restricted regression has been often applied to medical data (e.g., growth, toxicological, or pharmaceutical data), it has rarely been used for environmental data. Examples where this is done are Wu et al. (2001) (temperature anomalies), Leitenstorfer and Tutz (2007) (air pollution), and Tibshirani et al. (2011) (temperature anomalies).

Quantile regression is considered to be robust to outliers (Koenker 2005; Chandler and Scott 2011). Though trend estimates based on quantile regression are almost unbiased, they are still noisy for high quantiles owing to data scarcity. Therefore, it can be advantageous to consider a regional approach where the sitespecific quantiles exhibit a common relative trend. This common trend is generally more precisely estimated than the trends of the individual sites.

In this study, we propose a new algorithm for (regional) monotone regression of precipitation quantiles. This algorithm is used to explore the changes in the 0.95 , 0.975 , and 0.99 quantiles of daily precipitation in the Netherlands. The $p$ values are compared with those obtained from the (regional) MK test, proposed in Douglas et al. (2000). In section 2 we introduce the considered precipitation data. The methods are explained in section 3. In section 4 we present the results and the comparison with the MK test.

\section{Data}

The domain of the study is the Netherlands (NL) for which we have a high-quality dataset of observed daily precipitation sums for a long period. The data consist of 102 records of daily precipitation sums over the country from 1910 to 2009 that were corrected for inhomogeneities owing to changes in local measurement conditions (Buishand et al. 2013). The selected records exhibited at most $5 \%$ missing values. Zolina et al. (2008) showed that the effects of gaps on the trend estimates are practically negligible if less than $10 \%$ of the data are missing. Moreover, missing values were supplemented with the data from the nearest station, which is possible because the gradients in the percentiles of (extreme) daily rainfall are small in the Netherlands.

We apply the procedures locally and on the (sub) national level. The individual measurement stations and the considered subregions are shown in Fig. 1. A similar subdivision was applied in Beersma and Buishand (2007) for regional drought and Daniels et al. (2014) for trends in precipitation over the period 1950-2009.

Buishand et al. (2013) studied trends over the periods 1910-2009 and 1951-2009 for the Netherlands. Significant increases were found for annual precipitation and the precipitation amounts in the winter and summer

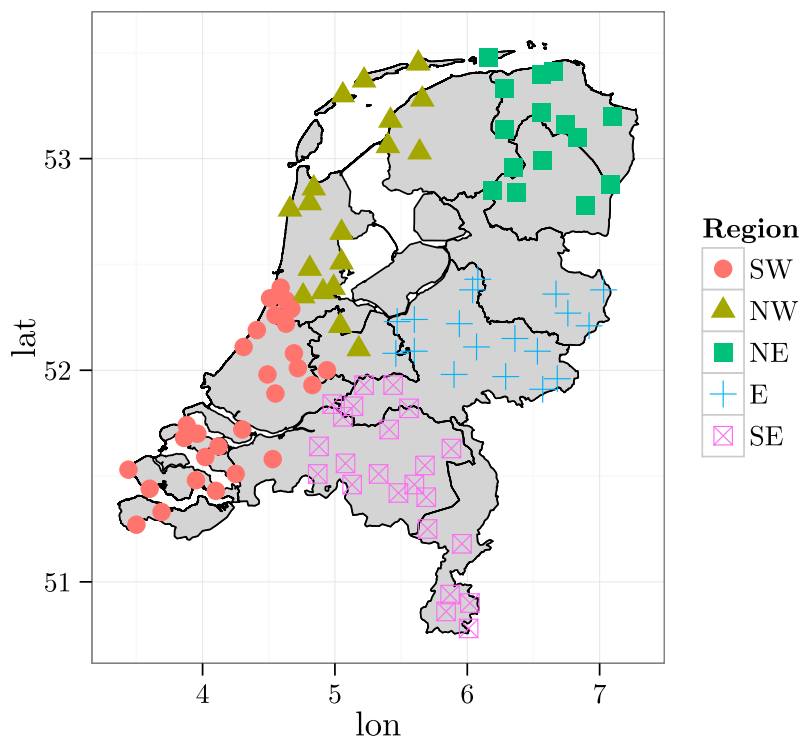

FIG. 1. The Netherlands with the considered stations by subregions.

halves of the year. The strongest increases were found in the winter season, which is consistent with the increases found in western and central Europe (Moberg and Jones 2005; Van den Besselaar et al. 2013). Significant increases in the summer season were mainly restricted to coastal regions. The latter are probably related to the increase in sea surface temperature (Lenderink et al. 2009). Buishand et al. (2013) also found a clear coastal effect in the number of days per year with a precipitation amount $>30 \mathrm{~mm}$. Daniels et al. (2014) studied the trends in seasonal mean precipitation and quantiles of the wet-day precipitation amounts for the period 1951-2009. It was found that zones based on distance to the coast gave a more consistent picture of precipitation changes over time than regions based on soil type, topography, or urbanization.

\section{Methods}

We are interested in the evolution of precipitation quantiles. A commonly used approach is to compute annual or seasonal quantiles and to assess the significance of the trend by the MK test for stationarity. The MK test is distribution free, thus the null distribution of the test statistic does not depend on the probability distribution from which the data are drawn. Moreover, it has high power against a one- or two-sided monotone trend alternative (Yue et al. 2002). In the following we first discuss the MK test before we explain the alternative monotone quantile regression method.

\section{a. Mann-Kendall test}

Consider a sequence of random variables $Z_{i}$, distributed according to $F_{i}(i=1, \ldots, n)$. In our application, 
$Z_{i}$ represents a sample quantile for year $i$. The MannKendall test uses the Kendall score $K$ [i.e., the difference between the number of concordant pairs $\left(Z_{j}>Z_{i}\right.$ for $\left.j>i\right)$ and the number of discordant pairs $\left(Z_{j}<Z_{i}\right.$ for $\left.\left.j>i\right)\right]$ to test the hypothesis that all distributions $F_{i}$ are equal. Annual and seasonal quantiles exhibit some autocorrelation at lags 1 and 2, which influences the distribution of $K$. To account for that we use a Monte Carlo permutation procedure for the computation of $p$ values, resampling blocks of $3 \mathrm{yr}$ (Carlstein 1986; Leander et al. 2014).

We follow Douglas et al. (2000) in their definition of an average MK statistic for testing the significance of the trend at the regional scale. For a region with $S$ time series we calculate first as above for each time series the statistic $K$ and denote it by $K_{s}$ for time series $s, 1 \leq s \leq S$. In the second step we consider the average of these: $\bar{K}_{S}=(1 / S) \sum_{s=1}^{S} K_{s}$. As in the univariate case we use a random permutation test for the computation of $p$ values. To account for the interdependence between samples, we resample spatiotemporal blocks of $3 \mathrm{yr}$ (i.e., all $S$ time series are permuted in the same way).

\section{b. Quantile regression}

\section{1) LINEAR QUANTILE REGRESSION}

The $\tau$ sample quantile of a dataset $y_{1}, \ldots, y_{T}$ (e.g., daily rainfall data) can be computed by minimizing the following objective function (Koenker 2005):

$$
\phi(\gamma):=\sum_{t=1}^{T} \rho_{\tau}\left(y_{t}-\gamma\right),
$$

where

$$
\rho_{\tau}(x)= \begin{cases}(\tau-1) x & x \leq 0 \\ \tau x & x>0\end{cases}
$$

The value of $\gamma$ for which $\phi$ is minimal is the estimator. Estimators of this type are known as M estimators. The M-estimator approach provides an alternative for the computation of quantiles, which are often computed using a sorting approach. Its main advantage comes to light when considering quantile regression. Analogously to linear least squares regression, we obtain a linear quantile regression by computing the following (Koenker 2005):

$$
\underset{\beta_{0}, \beta_{1} \in \mathbb{R}}{\arg \min } \sum_{t=1}^{T} \rho_{\tau}\left[y_{t}-\left(\beta_{0}+\beta_{1} t\right)\right] .
$$

For the median $(\tau=0.5)$ this is equivalent to the minimization of the sum of the absolute differences. Linear quantile regression has often been used; for example, for the determination of time-dependent thresholds in

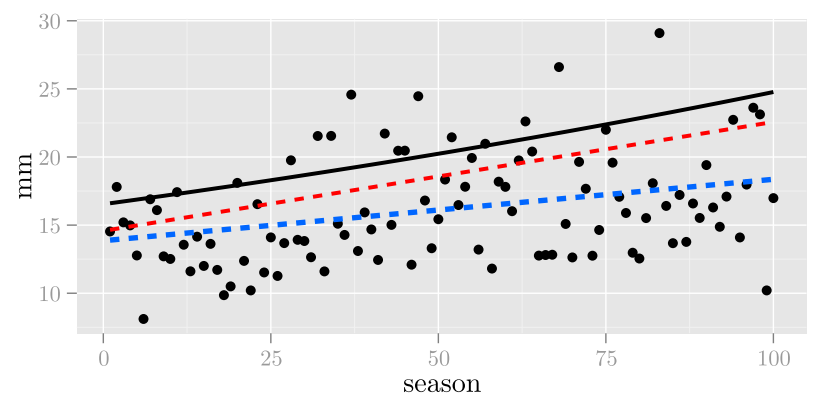

FIG. 2. Seasonal 0.99 quantiles (dots) and theoretical 0.99 quantile (solid black line) of simulated precipitation data. We simulated 100 days per season with each day having a $40 \%$ chance of being dry; otherwise, an exponentially distributed rainfall amount with mean increasing linearly from $4 \mathrm{~mm}$ at the beginning to $6 \mathrm{~mm}$ in the end. The dashed blue line gives the linear regression of the seasonal quantiles and the dashed red line shows the linear quantile regression.

peaks-over-threshold analysis (Kyselý et al. 2010), for summarizing trends in different parts of the distribution (Barbosa et al. 2011), and for scaling of extreme precipitation with temperature (Wasko and Sharma 2014).

So far the temporal evolution of a quantile is more often studied by first estimating annual quantiles and then performing a regression analysis based on these. The difference between quantile regression and the traditional approach of fitting a linear trend to seasonal quantiles is illustrated with synthetic precipitation data from an exponential distribution in Fig. 2. The figure shows that the linear regression of seasonal quantiles considerably underestimates the theoretical quantile. This is not the case for linear quantile regression.

\section{2) Monotone QuAntile Regression}

We consider monotone quantile regression (MQR) [i.e., we search a monotone regression function instead of the linear one in Eq. (3); see Casady and Cryer (1976)]. The optimization depends on the regression function only via its values at discrete $t$ s. Therefore, we consider only monotone piecewise constant functions and, hence, obtain the following optimization problem in the case of a nondecreasing trend:

$$
\underset{r_{1} \leq \cdots \leq r_{T}}{\arg \min } \sum_{t=1}^{T} \rho_{\tau}\left(y_{t}-r_{t}\right),
$$

where $r_{t}$ represents the value of the monotone function evaluated at time $t$. A nonincreasing trend can be estimated by transforming the data [e.g., a nonincreasing trend in the $\tau$ quantile is obtained as the negative nondecreasing trend in the $(1-\tau)$ quantile of $\left.-y_{t}\right]$. In this paper only nondecreasing trends are considered.

A common problem with monotone regression is the so-called spiking problem (i.e., the estimator is not 

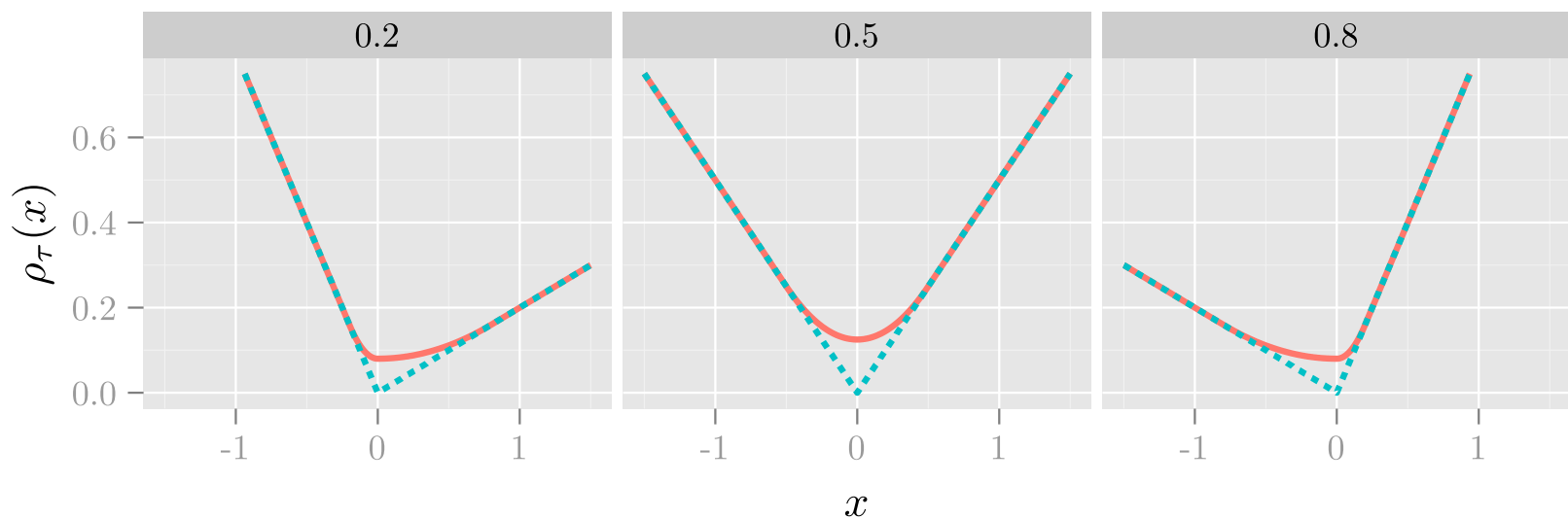

FIG. 3. The function $\rho_{\tau}$ (dashed blue line) and its smooth approximation (solid red line) for (left)-(right) $\tau=0.2,0.5$, and 0.8 with $c=1$ (after Muggeo et al. 2012).

consistent at the endpoints) (Pal 2008; Groeneboom and Jongbloed 2013), because the monotonicity constraint does not prevent the estimate from following the (erratic) behavior of the $y_{t}$ s near the boundary. Moreover, a smoother trend might be more suitable to visualize the changing behavior. To tackle the spiking problem and in order to obtain a smoother trend estimate we introduce a penalty on the second-order difference of the vector $\mathbf{r}=\left(r_{1}, \ldots, r_{T}\right) \quad$ (cf. Eilers and Marx 1996; Koenker 2005) and compute the following:

$$
\underset{r_{1} \leq \cdots \leq r_{T}}{\arg \min }\left[\sum_{t=1}^{T} \rho_{\tau}\left(y_{t}-r_{t}\right)+\lambda P(\mathbf{r})\right],
$$

where

$$
P(\mathbf{r}):=\sum_{t=2}^{T-1}\left(r_{t+1}-2 r_{t}+r_{t-1}\right)^{2}
$$

is the sum of the squared second-order differences. By introducing this penalty, variations at the endpoints are not free anymore as larger parts of $\mathbf{r}$ will be affected. Moreover, for $\lambda \rightarrow \infty$ the solution of the optimization problem in Eq. (5) will become linear (any deviation from linearity would lead to an immense increase in the second term). An alternative approach would be to use a penalty on the total variation (Groeneboom and Jongbloed 2013), which is in the monotonic setting equivalent to the difference between the endpoints, and smooth the obtained estimator using, for example, kernel smoothing (Wand and Jones 1994).

\section{3) Computational issues}

Koenker and $\mathrm{Ng}$ (2005) use an interior point algorithm to fit a smooth monotone quantile regression using splines and a penalty on the total variation. Bollaerts et al. (2006) approach the same problem with a slightly different $P$-splines method, allowing for nearly monotone trends, by introducing a penalty on monotonicity violations.

The fact that $\rho_{\tau}$ is not differentiable, in contrast to the sum-of-squares loss function, hinders the use of standard gradient based optimization techniques, such as the Newton-Raphson method.

Recently Muggeo et al. (2012), following others, proposed a slight modification of $\rho_{\tau}$, namely,

$\tilde{\rho}_{\tau}^{(c)}(x)=\left\{\begin{array}{lll}(\tau-1) x & \text { if } \quad x \leq-c \tau, \\ \frac{(1-\tau) x^{2}}{2 c \tau}+\frac{c \tau(1-\tau)}{2} & \text { if } \quad-c \tau<x \leq 0, \\ \frac{\tau x^{2}}{2 c(1-\tau)}+\frac{c \tau(1-\tau)}{2} & \text { if } & 0<x<c(1-\tau), \\ \tau x & \text { if } \quad x \geq c(1-\tau),\end{array}\right.$

see Fig. 3. This differentiable approximation of $\rho_{\tau}$ facilitates the use of other optimization techniques than interior point methods. Therefore, we consider the following optimization problem:

$$
\underset{r_{1} \leq \cdots \leq r_{T}}{\arg \min } \phi_{\lambda}\left(r_{1}, \ldots, r_{T}\right),
$$

where

$$
\phi_{\lambda}\left(r_{1}, \ldots, r_{T}\right):=\sum_{t=1}^{T} \tilde{\rho}_{\tau}^{(c)}\left(y_{t}-r_{t}\right)+\lambda P(\mathbf{r})
$$

Muggeo et al. (2012) state that the choice of $c$ has only limited impact on the final estimates because of the special form of the approximation. In the rest of the article, 
we consider only a fixed value of 0.1 for $c$. The monotonicity constraint in the optimization problem in Eq. (8) is tackled with the iterative convex minorant algorithm, see the appendix.

When we are interested in the estimation of the longterm trend the seasonal cycle is not relevant. Hence, the dimensionality of the optimization problem can be reduced by specifying that the quantile estimates should be constant over the year or season. Instead of the $T$-dimensional vector $\mathbf{r}$ we consider an $n$-dimensional vector $\tilde{\mathbf{r}}=\left(\tilde{r}_{1}, \ldots, \tilde{r}_{n}\right)$, with $n$ denoting the number of years. Owing to the dimension reduction we need less iterations of the algorithm and, hence, less computation time.

\section{4) SMoothing PENALty}

A difficulty in nonparametric frameworks is often the selection of the smoothing penalty. Koenker (2005) presents a modified version of the Schwarz-Bayesian information criterion based on counting the pieces of a piecewise linear fit. However, our fit is not piecewise linear and, thus, this approach cannot be applied. A possible way to determine the optimal smoothing parameter in this situation is by cross validation, using the quantile verification score, which is the value of the objective function $\phi$ in Eq. (1) evaluated for the independent data at the prediction $\hat{\gamma}$ (Gneiting and Raftery 2007; Friederichs and Hense 2007).

\section{5) Regional MONOTONE QUANTILE REGRESSION}

Apart from local trends we would like to estimate a common trend over a region, which we expect to have smaller standard error than the local trend estimates. The common trend in the $\tau$ quantile is estimated after scaling the daily rainfall amounts by the site-specific sample $\tau$ quantile $\hat{q}_{s}(\tau)$. Analogously as in the univariate case this trend is obtained as

$$
\underset{r_{1} \leq \cdots \leq r_{T}}{\arg \min }\left\{\sum_{s=1}^{S} \sum_{t=1}^{T} \tilde{\rho}_{\tau}^{(c)}\left[\frac{y_{s, t}}{\hat{q}_{s}(\tau)}-r_{t}\right]+\lambda P(\mathbf{r})\right\},
$$

where $y_{s, t}$ is the daily rainfall amount at site $s \in\{1, \ldots, S\}$ and day $t \in\{1, \ldots, T\}$. Note that $\mathbf{r}$ now represents a common trend in the standardized $\tau$ quantile and that $r_{t}=1$, for all $t$ in the case of no trend.

\section{6) Testing}

MQR can be used to test the null hypothesis that the quantile of interest is constant over time:

$$
H_{0}: \tilde{r}_{i} \equiv \beta
$$

against the alternative that it is nondecreasing:

$$
H_{1}: \tilde{r}_{1} \leq \cdots \leq \tilde{r}_{n} .
$$

As for the MK test we use a Monte Carlo permutation test for the calculation of $p$ values, preserving the spatiotemporal correlation structure of the data. We evaluate the resampled data by calculating the $l_{2}$ difference between the constant quantile and the monotone fit. Taking the $l_{1}$ difference or the difference between the minima of the objective criteria in Eqs. (1) and (4) gives very similar results.

An advantage of the MQR over the MK approach is that we can also test the null hypothesis that the quantile is increasing linearly over time:

$$
H_{0}^{*}: \tilde{r}_{i}=\alpha_{0}+\alpha_{1} i, \quad \alpha_{1}>0,
$$

versus the more flexible one-sided monotone trend alternative $H_{1}$. To apply also here a permutation type test we have to generate appropriate data samples satisfying the null hypothesis. Therefore, we compute the residuals $z_{t}=y_{t}-r_{t}$, where $r_{t}=\hat{r}_{i}^{H_{0}^{*}}$ if day $t$ falls in year $i$, permute these as before in 3-yr blocks, and add then $r_{t}$. As a test statistic, we use the $l_{2}$ difference between the estimated linear trend $\tilde{\mathbf{r}}^{H_{0}^{*}}$ and the nondecreasing trend $\tilde{\mathbf{r}}^{H_{1}}$.

\section{Results}

\section{a. Local application}

For each of the five regions in Fig. 1 we selected one station and applied cross validation, using the quantile verification score (Friederichs and Hense 2007), to determine the smoothing parameter $\lambda$. In the cross validation we skipped a block of one year or season of the data and estimated the nondecreasing trend for a given $\lambda$, based on the remaining data. The prediction $\hat{\gamma}$ for the skipped block was then defined as the average of the estimated values for the neighboring blocks. Using this prediction, we computed the quantile verification score for the independent data (i.e., the skipped block). This was done for every block, leading to 100 prediction scores for the given smoothing parameter. The procedure was repeated for several smoothing parameters $\lambda \in[0, \infty]$, where $\lambda=\infty$ refers to linear estimation restricted to nonnegative slope estimates.

The prediction performances for the considered smoothing parameters are very similar and the optimal parameter varies over stations and quantiles. For some combinations the optimal parameter is quite small and for others it is very large. In general we can say that the differences in the mean predictive score for different 


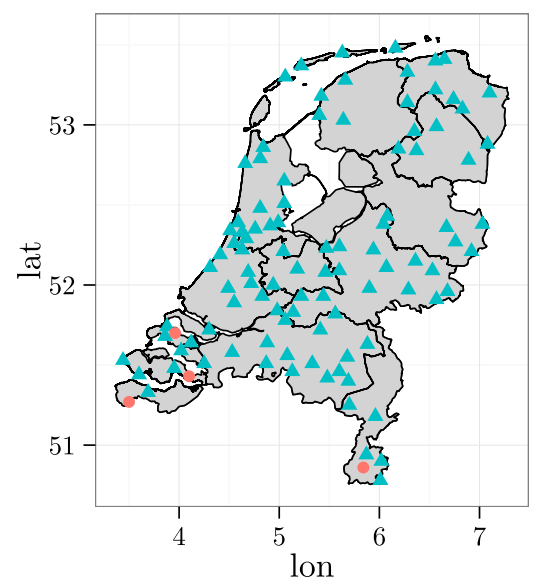

(a) Year

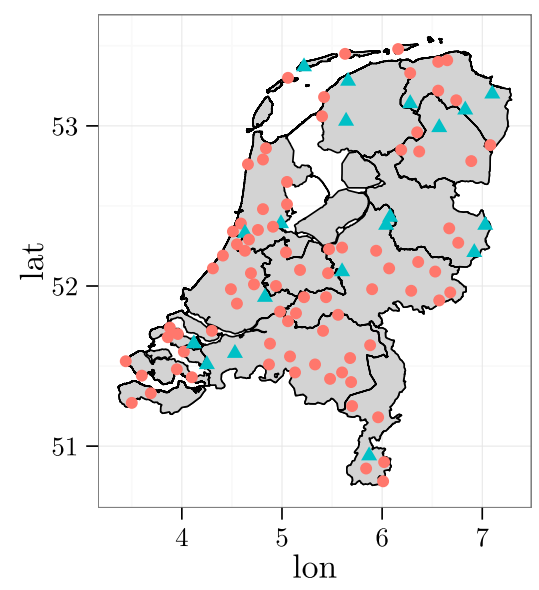

(b) Summer

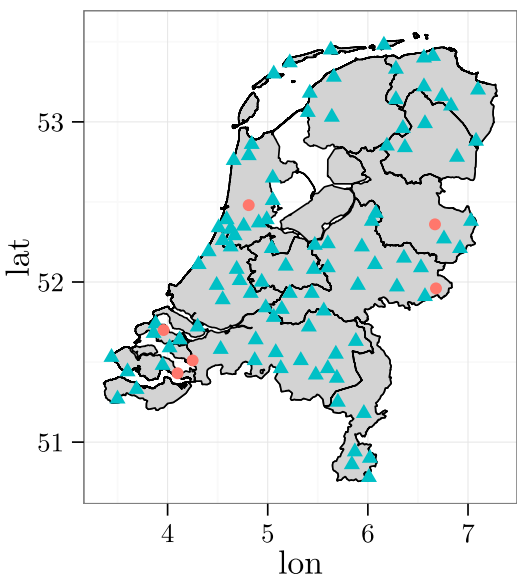

(c) Winter

FIG. 4. Stations with significant positive trend in the 0.95 quantile (blue triangles) and nonsignificant trend (red dots) at the $5 \%$ level, as obtained by MQR: (a)-(c) year, summer, and winter.

smoothing parameters are very small compared to the annual variation of the score for the optimal parameter. Because of the inconsistent picture for the data at hand and the increased computational burden for large smoothing parameters, we decided to consider a relatively small, fixed value of $\lambda$ (300) for all sites and quantiles.

Then we tested the null hypothesis of no trend in the 0.95 quantile versus the alternative of a nondecreasing trend for each of the 102 stations. Figure 4 shows the results of the test at the 5\% significance level for the annual, winter (December-February), and summer (June-August) 0.95 quantiles. While in summer we only have a few significant trends, in winter an overwhelming majority of the sites shows a significant increase. For the whole year most stations also exhibit a significant increase, which can be expected given the increase in the winter season. In general this is in line with earlier work about trends in extreme precipitation in the Netherlands, as discussed in section 2. For comparison the spatial distribution of significant trends obtained by the MK test is shown in Fig. 5. The differences between the MQR and the MK approach are small for winter and annual quantiles. In summer the differences are somewhat larger. There are more significant trends detected by the MQR framework than by the MK test, but the spatial distribution of stations with a significant trend is more scattered.

The relative change over the period 1910-2009, with respect to the estimate for 1910, was spatially interpolated over the Netherlands, using simple kriging

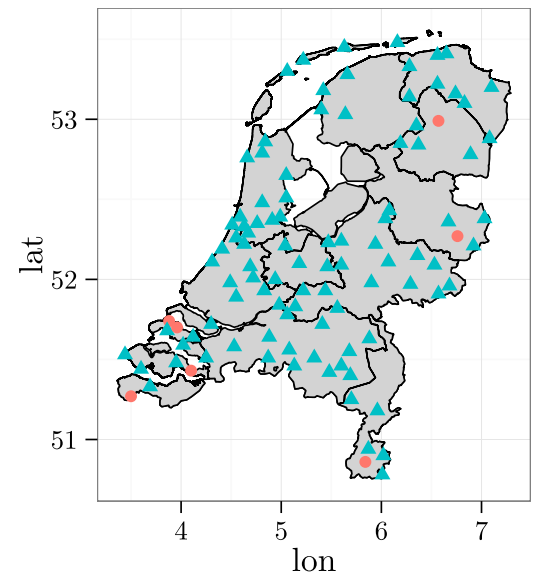

(a) Year

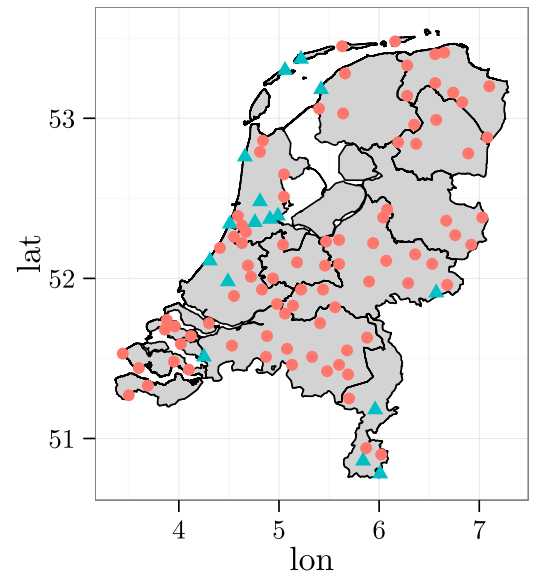

(b) Summer

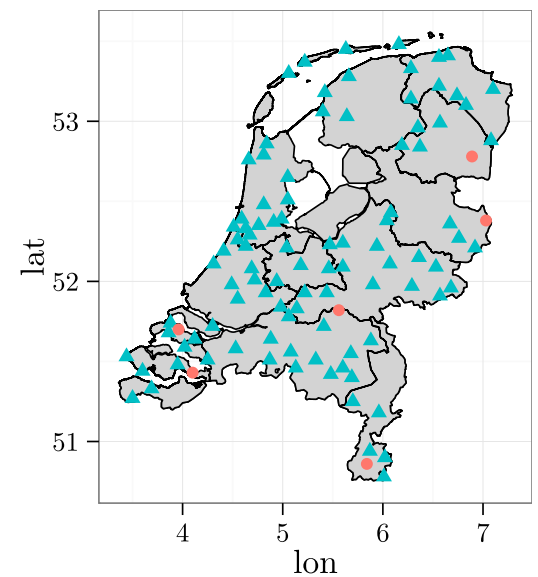

(c) Winter

FIG. 5. As in Fig. 4, but for the MK test. 


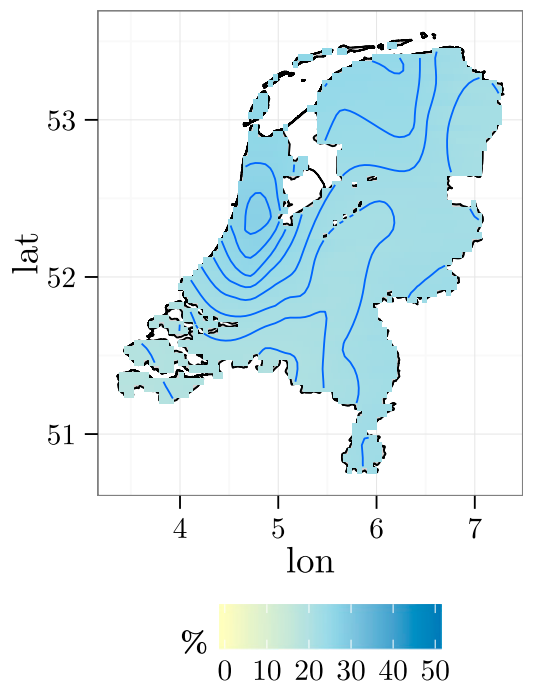

(a) Year

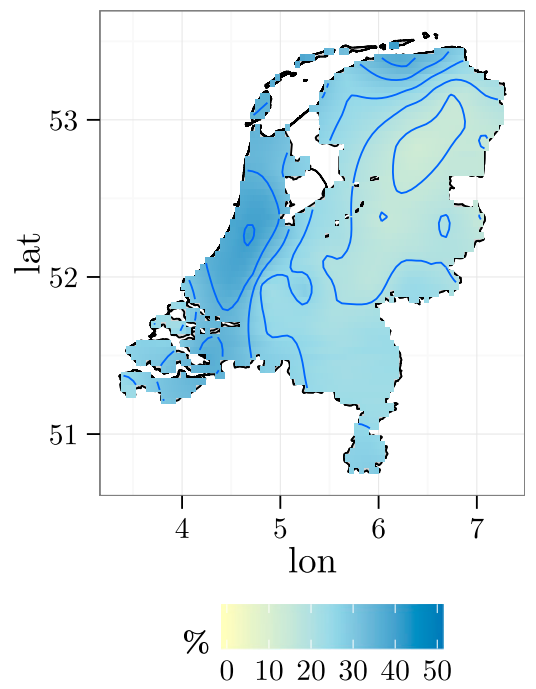

(b) Summer

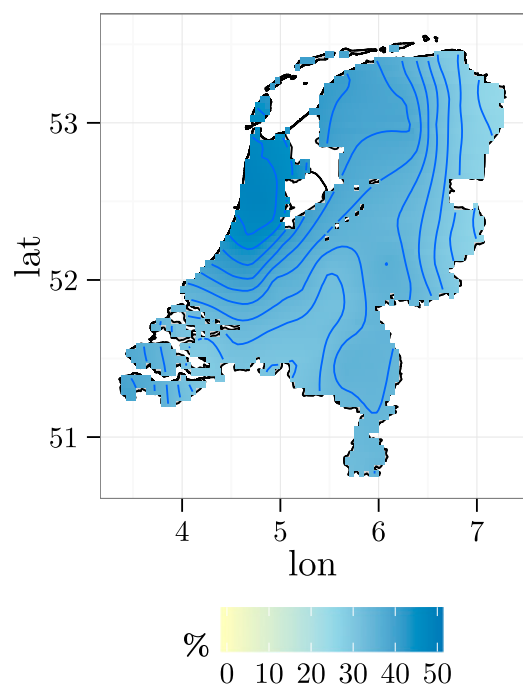

(c) Winter

FIG. 6. Relative increase (\%) in the 0.95 quantile: (a)-(c) year, summer, and winter.

(Gaetan and Guyon 2010). Figures 6, 7, and 8 show the relative increase in the $0.95,0.975$, and 0.99 quantiles, respectively. In summer, we observe a clear coastal effect in the west of the country, which is strongest in the 0.99 quantile. A somewhat weaker coastal effect is also visible in winter, where we do not observe any quantile dependence of the effect. For the annual values the coastal effect is less pronounced and cannot be observed on the color scale used. The stronger the deviation from linearity the more sensitive is the magnitude of the trend to the choice of the smoothing parameter. In particular, the results for the 0.99 summer quantile turned out to be affected by this choice.

\section{b. Regional application}

The smoothing parameters for the different regions and quantiles were chosen after a similar consideration as in the local setting. Table 1 shows the relative change over the century for the whole NL and the subregions defined in section 2 . The relative change is considerably larger in winter than in summer. Moreover, while the relative change in winter is about the same for the three

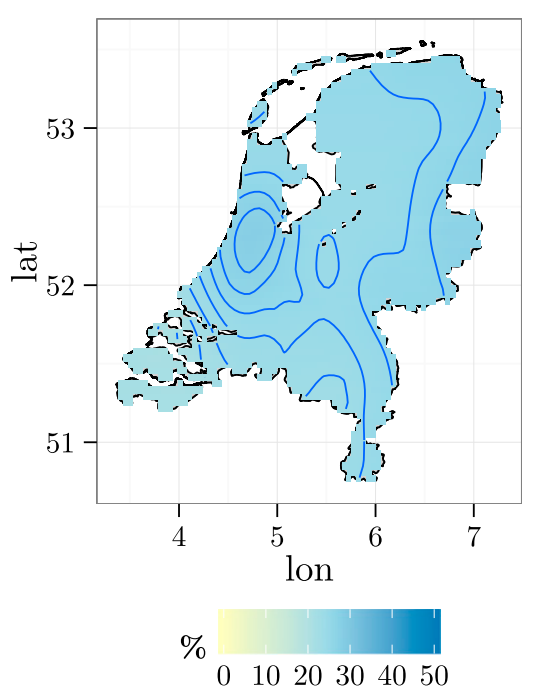

(a) Year

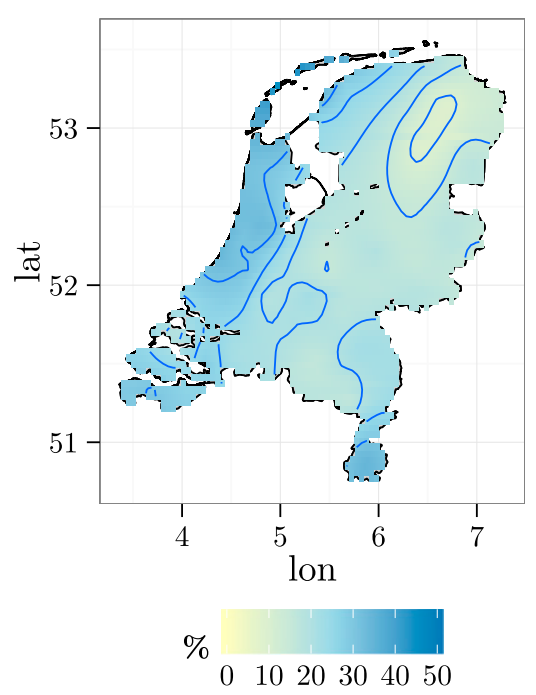

(b) Summer

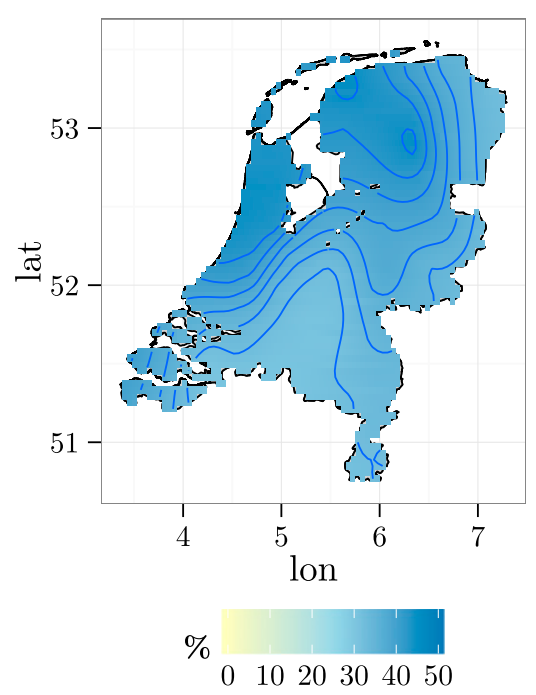

(c) Winter

FIG. 7. As in Fig. 6, but for the 0.975 quantile. 


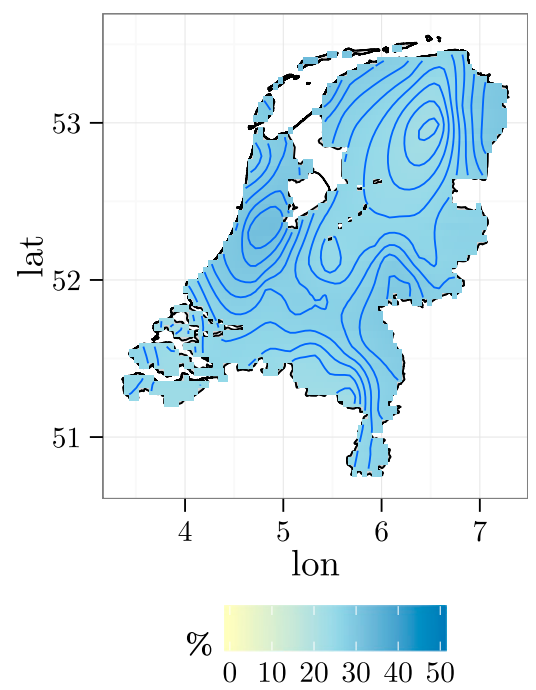

(a) Year

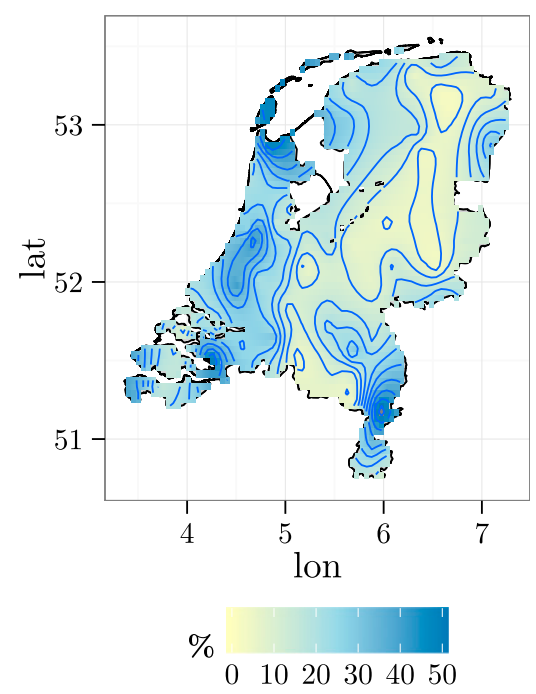

(b) Summer

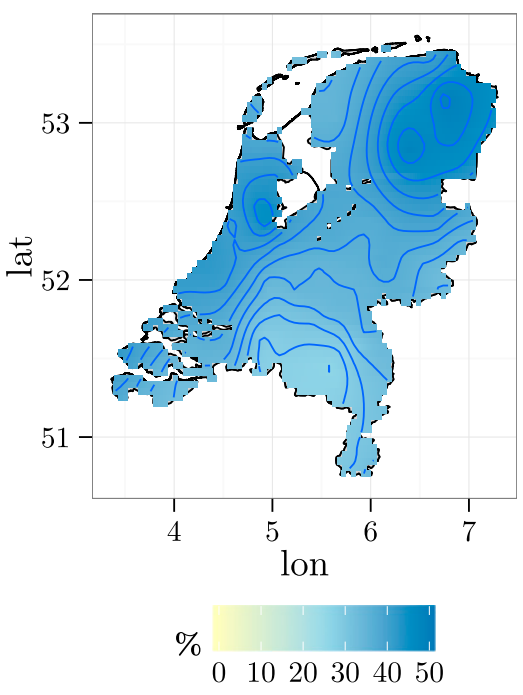

(c) Winter

FIG. 8. As in Fig. 6, but for the 0.99 quantile.

quantiles it is decreasing for higher quantiles in the summer season and for the whole year. Table 2 shows the corresponding $p$ values of the regional test for stationarity using the MQR approach. We see that all regions show a significant increase at the 5\% level for the annual and winter quantiles. In summer we obtain $p$ values mostly below 0.05 for both western regions and mostly below 0.1 for the southeast region as well as for the whole Netherlands. The results obtained from the regional MK test are quite similar. In fact, only 1 (5) out of the 36 combinations of season, quantile, and region give different results at the $10 \%$ (5\%) significance level.

Figure 9 shows the trend in the standardized 0.99 quantile with a pointwise confidence band for the northwest (NW) region in winter. Although we clearly see two plateaus in the 1920s and late 1960s, no large deviation from linearity can be detected, and indeed the trend in the standardized quantile is almost the same as for a

TABLE 1. Relative increase in the $\tau$ quantile in percent as obtained from MQR.

\begin{tabular}{llllllll}
\hline \hline \multicolumn{1}{c}{$\tau$} & Season & NL & SW & NW & NE & E & SE \\
\hline 0.99 & Summer & 22.1 & 42.2 & 28.6 & 11.7 & 9.0 & 14.6 \\
0.975 & Summer & 27.2 & 31.1 & 31.1 & 16.2 & 13.5 & 30.0 \\
0.95 & Summer & 38.5 & 43.8 & 37.4 & 22.7 & 23.0 & 40.9 \\
0.99 & Winter & 37.3 & 44.7 & 39.1 & 46.2 & 44.1 & 32.8 \\
0.975 & Winter & 37.0 & 45.6 & 41.7 & 37.8 & 36.7 & 32.6 \\
0.95 & Winter & 43.5 & 46.0 & 43.1 & 29.6 & 32.7 & 39.6 \\
0.99 & Year & 28.8 & 27.9 & 27.7 & 25.9 & 23.7 & 26.3 \\
0.975 & Year & 33.2 & 32.0 & 27.7 & 26.7 & 34.5 & 32.9 \\
0.95 & Year & 37.2 & 36.4 & 38.5 & 32.4 & 41.2 & 34.4 \\
\hline
\end{tabular}

linear fit. Figure 10 shows the standardized 0.99 quantile in summer, where the overall trend is smaller than in winter. Moreover, linearity of the trend seems less clear than in winter and the relative change from MQR is larger than for the linear fit. However, the linearity test described in section 3 did not reject linearity at the $5 \%$ level and for larger smoothing parameters the trend becomes more linear again.

\section{Conclusions}

With the presented MQR approach we provide an additional tool to test the assumption of increasing rainfall extremes. MQR represents a unified method for visualizing nondecreasing trend behavior and testing the stationary hypothesis against the one-sided alternative of a monotone trend. The method can be used for local quantile regression as well as for the

TABLE 2. Resulting $p$ values in percent for the MQR test of the stationary hypothesis vs the one-sided monotone alternative.

\begin{tabular}{lllllllr}
\hline \hline \multicolumn{1}{c}{$\tau$} & Season & NL & SW & NW & NE & E & SE \\
\hline 0.99 & Summer & 8.6 & 2.2 & 1.1 & 32.0 & 33.5 & 22.7 \\
0.975 & Summer & 9.2 & 5.8 & 2.2 & 47.6 & 22.6 & 7.2 \\
0.95 & Summer & 4.3 & 4.9 & 2.5 & 18.0 & 24.0 & 5.2 \\
0.99 & Winter & 0 & 0 & 0 & 0 & 1.1 & 0.2 \\
0.975 & Winter & 0 & 0 & 0 & 0 & 0.8 & 0.4 \\
0.95 & Winter & 0 & 0 & 0 & 0 & 0.8 & 0.4 \\
0.99 & Year & 0 & 0 & 0 & 0 & 0.1 & 0.1 \\
0.975 & Year & 0 & 0.1 & 0 & 0 & 0 & 0 \\
0.95 & Year & 0 & 0.1 & 0 & 0 & 0.1 & 0.1 \\
\hline
\end{tabular}




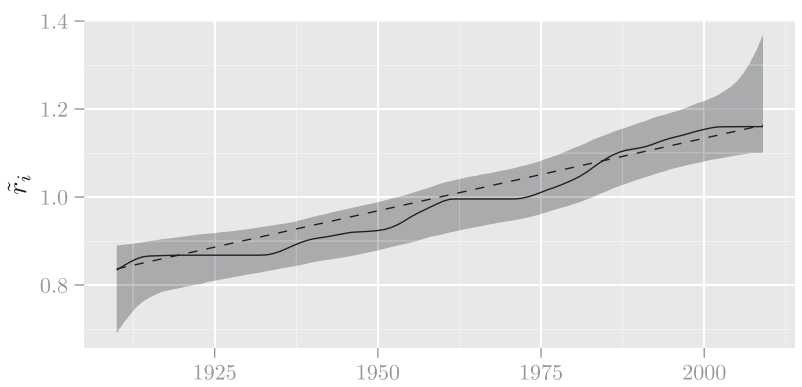

FIG. 9. Monotone (solid) and linear (dashed) trend in the standardized 0.99 quantile in winter (NW region). The gray area indicates a pointwise $95 \%$ confidence band for the monotone trend.

estimation of a common regional trend, given sitespecific quantiles. Similar results regarding the significance of trends were obtained with MQR and the MK test, both in the local and regional application. Unlike the MK test, MQR also visualizes the trend. Furthermore, MQR does not rely on annual quantiles that might be substantially biased and allows for additional testing of the trend structure (e.g., nonlinear versus linear behavior). However, while the algorithm for local and regional monotone quantile regression works fast, it is computationally more demanding than the calculation of the Kendall score. Therefore, Monte Carlo experiments take considerably more time.

Our analysis strengthens the assumption that the annual and winter rainfalls have increased throughout the Netherlands. In summer, the behavior is subject to a coastal effect as previously discovered by Lenderink et al. (2009) and also supported here [see also Daniels et al. (2014)]. Trends in summer precipitation extremes were not significant in the eastern part of the country, which is in agreement with the results of Zolina et al. (2008) for precipitation extremes during the period 1950-2004 for the adjacent German region. In other German regions, many stations showed a significant negative trend in summer extremes during that period. The increase in summer extremes in the Netherlands seems to accelerate in recent years; however, a significant deviation from linearity could not be detected. This finding supports the widespread use of linear trends in the analysis of high precipitation quantiles. Buishand et al. (2013) detected a significant deviation from linearity in the trend of the spatially averaged annual number of days exceeding 30-mm rainfall over the Netherlands. This is not in contradiction to the findings reported here, because $30 \mathrm{~mm}$ represents a higher quantile than considered in the study at hand (in the number of days exceeding $20 \mathrm{~mm}$ no significant nonlinearity was found). However, it emphasizes the need for continued attention regarding nonlinearity.

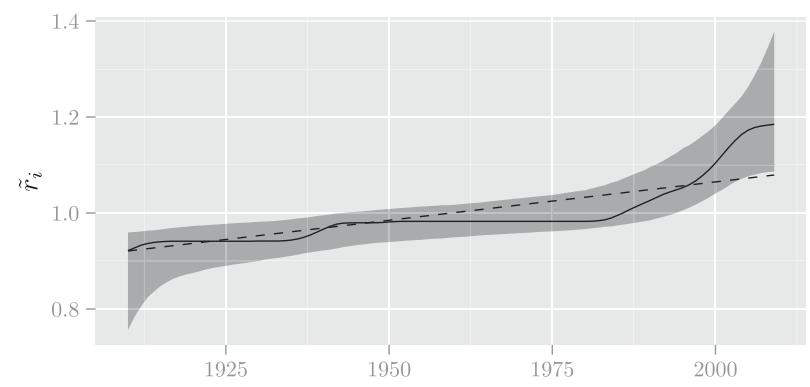

FIG. 10. As in Fig. 9, but for the summer season.

Acknowledgments. The research was supported by the Dutch research program Knowledge for Climate. The unpublished dataset developed in Buishand et al. (2013) was kindly made available by T. Brandsma. All calculations were performed using the R environment (http:// www.r-project.org). We are grateful to three reviewers for their helpful comments.

\section{APPENDIX}

\section{Iterative Convex Minorant Algorithm}

The iterative convex minorant algorithm is based on minimizing successive approximations of the objective function $\phi_{\lambda}$ in Eq. (8). To be more specific, for a given iterate $\mathbf{r}^{(k)}=\left[r_{1}^{(k)}, \ldots, r_{T}^{(k)}\right]$ we consider the following optimization problem:

$$
\underset{r_{1} \leq \cdots \leq r_{T}}{\arg \min } \sum_{t=1}^{T}\left\{r_{t}-r_{t}^{(k)}+\frac{\partial \phi_{\lambda}}{\partial r_{t}}\left[\mathbf{r}^{(k)}\right]\right\}^{2} .
$$

The unique solution of this problem can be easily constructed, using the theory of order restricted regression (Robertson et al. 1988). First define the cumulative sum diagram consisting of the points $P_{0}=(0,0)$ and

$$
P_{t}=\left\langle t, \sum_{j=1}^{t}\left\{r_{j}^{(k)}-\frac{\partial \phi_{\lambda}}{\partial r_{j}}\left[\mathbf{r}^{(k)}\right]\right\}\right\rangle,
$$

for $1 \leq t \leq T$. Then construct the (greatest) convex minorant of these points. The left derivative of the convex minorant evaluated at point $P_{t}$ is denoted by $\mathbf{r}_{t}^{\text {New }}$. Compared to $\mathbf{r}^{(k)}$ the new vector $\mathbf{r}^{\mathrm{New}}$ is a step in the right direction (i.e., of decreasing $\phi_{\lambda}$ ); however, it might be too big $\left\{\right.$ i.e., $\left.\phi_{\lambda}\left(\mathbf{r}^{\text {New }}\right)>\phi_{\lambda}\left[\mathbf{r}^{(k)}\right]\right\}$. Therefore, we obtain the next iterate as a convex combination of $\mathbf{r}^{(k)}$ and $\mathbf{r}^{\text {New}}$ :

$$
\mathbf{r}^{(k+1)}=\mathbf{r}^{(k)}+u\left[\mathbf{r}^{\mathrm{New}}-\mathbf{r}^{(k)}\right],
$$

for some $u \in(0,1]$, such that $\phi_{\lambda}\left[\mathbf{r}^{(k+1)}\right]<\phi_{\lambda}\left[\mathbf{r}^{(k)}\right]$. As a starting value for the algorithm we use the constant 
sample quantile. For more information on the iterative convex minorant algorithm see Jongbloed (1998).

\section{REFERENCES}

Alexander, L. V., and Coauthors, 2006: Global observed changes in daily climate extremes of temperature and precipitation. J. Geophys. Res., 111, D05109, doi:10.1029/2005JD006290.

Barbosa, S. M., M. G. Scotto, and A. Alonso, 2011: Summarising changes in air temperature over Central Europe by quantile regression and clustering. Nat. Hazards Earth Syst. Sci., 11, 3227-3233, doi:10.5194/nhess-11-3227-2011.

Beersma, J. J., and T. A. Buishand, 2007: Drought in the NetherlandsRegional frequency analysis versus time series simulation. J. Hydrol., 347, 332-346, doi:10.1016/j.jhydrol.2007.09.042.

Bollaerts, K., P. H. C. Eilers, and M. Aerts, 2006: Quantile regression with monotonicity restrictions using P-splines and the $L_{1}$-norm. Stat. Model., 6, 189-207, doi:10.1191/1471082X06st118oa.

Buishand, T. A., G. De Martino, J. Spreeuw, and T. Brandsma, 2013: Homogeneity of precipitation series in the Netherlands and their trends in the past century. Int. J. Climatol., 33, 815833, doi:10.1002/joc.3471.

Carlstein, E., 1986: The use of subseries values for estimating the variance of a general statistic from a stationary sequence. Ann. Stat., 14, 1171-1179, doi:10.1214/aos/1176350057.

Casady, R. J., and J. Cryer, 1976: Monotone percentile regression. Ann. Stat., 4, 532-541, doi:10.1214/aos/1176343459.

Chandler, R., and M. Scott, 2011: Statistical Methods for Trend Detection and Analysis in the Environmental Sciences. Wiley, $388 \mathrm{pp}$.

Daniels, E. E., G. Lenderink, R. W. A. Hutjes, and A. A. M. Holtslag, 2014: Spatial precipitation patterns and trends in the Netherlands during 1951-2009. Int. J. Climatol., 34, 1773-1784, doi:10.1002/joc.3800.

Douglas, E., R. Vogel, and C. Kroll, 2000: Trends in floods and low flows in the United States: Impact of spatial correlation. J. Hydrol., 240, 90-105, doi:10.1016/S0022-1694(00)00336-X.

Eilers, P. H. C., and B. D. Marx, 1996: Flexible smoothing with B-splines and penalties. Stat. Sci., 11, 89-102, doi:10.1214/ss/ 1038425655.

Friederichs, P., and A. Hense, 2007: Statistical downscaling of extreme precipitation events using censored quantile regression. Mon. Wea. Rev., 135, 2365-2378, doi:10.1175/MWR3403.1.

Gaetan, C., and X. Guyon, 2010: Spatial Statistics and Modeling. Springer, $302 \mathrm{pp}$.

Gneiting, T., and A. E. Raftery, 2007: Strictly proper scoring rules, prediction, and estimation. J. Amer. Stat. Assoc., 102, 359-378, doi:10.1198/016214506000001437.

Groeneboom, P., and G. Jongbloed, 2013: Smooth and non-smooth estimates of a monotone hazard. From Probability to Statistics and Back: High-Dimensional Models and Processes-A Festschrift in Honor of Jon A. Wellner, M. Banerjee et al., Eds., Institute of Mathematical Statistics, 174-196, doi:10.1214/ 12-IMSCOLL913.

Hartmann, D. L., and Coauthors, 2013: Observations: Atmosphere and surface. Climate Change 2013: The Physical Science Basis, T. F. Stocker et al., Eds., Cambridge University Press, 159-254.

Jongbloed, G., 1998: The iterative convex minorant algorithm for nonparametric estimation. J. Comput. Graph. Stat., 7, 310-321, doi:10.2307/1390706.

Klein Tank, A. M. G., and G. P. Können, 2003: Trends in indices of daily temperature and precipitation extremes in Europe, 1946-99.
J. Climate, 16, 3665-3680, doi:10.1175/1520-0442(2003)016<3665: TIIODT $>2.0 . \mathrm{CO} ; 2$

Koenker, R., 2005: Quantile Regression. Cambridge University Press, $366 \mathrm{pp}$.

, and P. Ng, 2005: Inequality constrained quantile regression. Sankhya, 67 (2), 418-440.

Kyselý, J., J. Picek, and R. Beranová, 2010: Estimating extremes in climate change simulations using the peaks-over-threshold method with a non-stationary threshold. Global Planet. Change, 72, 55-68, doi:10.1016/j.gloplacha.2010.03.006.

Leander, R., T. A. Buishand, and A. M. G. Klein Tank, 2014: An alternative index for the contribution of precipitation on very wet days to the total precipitation. J. Climate, 27, 1365-1378, doi:10.1175/JCLI-D-13-00144.1.

Leitenstorfer, F., and G. Tutz, 2007: Generalized monotonic regression based on B-splines with an application to air pollution data. Biostatistics, 8, 654-673, doi:10.1093/biostatistics/kxl036.

Lenderink, G., E. van Meijgaard, and F. Selten, 2009: Intense coastal rainfall in the Netherlands in response to high sea surface temperatures: Analysis of the event of August 2006 from the perspective of a changing climate. Climate Dyn., 32, 19-33, doi:10.1007/s00382-008-0366-x.

Moberg, A., and P. D. Jones, 2005: Trends in indices for extremes in daily temperature and precipitation in central and western Europe, 1901-99. Int. J. Climatol., 25, 1149-1171, doi:10.1002/ joc.1163.

Muggeo, V. M., M. Sciandra, and L. Augugliaro, 2012: Quantile regression via iterative least squares computations. J. Stat. Comput. Simul., 82, 1557-1569, doi:10.1080/00949655.2011.583650.

Pal, J. K., 2008: Spiking problem in monotone regression: Penalized residual sum of squares. Stat. Probab. Lett., 78, 1548-1556, doi:10.1016/j.spl.2008.01.015.

Robertson, T., F. T. Wright, and R. L. Dykstra, 1988: Order Restricted Statistical Inference. John Wiley \& Sons, 544 pp.

Tibshirani, R. J., H. Hoefling, and R. Tibshirani, 2011: Nearlyisotonic regression. Technometrics, 53, 54-61, doi:10.1198/ TECH.2010.10111.

Van den Besselaar, E. J. M., A. M. G. Klein Tank, and T. A. Buishand, 2013: Trend in European precipitation extremes over 1951-2010. Int. J. Climatol., 33, 2682-2689, doi:10.1002/ joc.3619.

Villarini, G., J. A. Smith, M. L. Baeck, R. Vitolo, D. B. Stephenson, and W. F. Krajewski, 2011: On the frequency of heavy rainfall for the Midwest of the United States. J. Hydrol., 400, 103-120, doi:10.1016/j.jhydrol.2011.01.027.

Wand, M. P., and M. C. Jones, 1994: Kernel Smoothing. Chapman \& Hall, $224 \mathrm{pp}$.

Wasko, C., and A. Sharma, 2014: Quantile regression for investigating scaling of extreme precipitation with temperature. Water Resour. Res., 50, 3608-3614, doi:10.1002/ 2013 WR015194.

Wu, W. B., M. Woodroofe, and G. Mentz, 2001: Isotonic regression: Another look at the changepoint problem. Biometrika, 88, 793-804, doi:10.1093/biomet/88.3.793.

Yue, S., P. Pilon, and G. Cavadias, 2002: Power of the MannKendall and Spearman's rho tests for detecting monotonic trends in hydrological series. J. Hydrol., 259, 254-271, doi:10.1016/S0022-1694(01)00594-7.

Zolina, O., C. Simmer, A. Kapala, S. Bachner, S. Gulev, and H. Maechel, 2008: Seasonally dependent changes of precipitation extremes over Germany since 1950 from a very dense observational network. J. Geophys. Res., 113, D06110, doi:10.1029/2007JD008393. 